Cahiers d'études italiennes

\title{
Guerre, politique et morale dans deux romans de Claudio Magris et Carlo Sgorlon
}

Pierre Laroche

\section{(2) OpenEdition}

\section{Journals}

Édition électronique

URL : http://journals.openedition.org/cei/291

DOI : 10.4000/cei.291

ISSN : 2260-779X

Éditeur

UGA Éditions/Université Grenoble Alpes

\section{Édition imprimée}

Date de publication : 15 juin 2005

Pagination : 223-234

ISBN : 978-2-84310-066-6

ISSN : 1770-9571

\section{Référence électronique}

Pierre Laroche, "Guerre, politique et morale dans deux romans de Claudio Magris et Carlo Sgorlon », Cahiers d'études italiennes [En ligne], 3 | 2005, mis en ligne le 15 décembre 2006, consulté le 26 mars 2021. URL : http://journals.openedition.org/cei/291 ; DOI : https://doi.org/10.4000/cei.291 


\title{
GUERRE, POLITIQUE ET MORALE \\ dans deux romans de Claudio Magris et Carlo Sgorlon
}

\author{
Pierre Laroche
}

Université de la Sorbonne nouvelle - Paris 3

À moins d'un an d'intervalle (1984 et 1985), Claudio Magris puis Carlo Sgorlon publient chacun un roman ${ }^{1}$ situé en Carnie, en 1944-1945, à l'époque où cette région était occupée par des troupes cosaques, alliées de l'Allemagne nazie avec laquelle ils n'avaient en commun que la haine de la Russie soviétique. Les nazis avaient recruté ces Cosaques ${ }^{2}$ en leur promettant la création d'un Kosakenland, qui aurait dû être situé sur le territoire de l'Union soviétique après la défaite de celle-ci ; l'évolution du conflit avait amené cette proposition à évoluer vers l'ouest, jusqu'au moment où cette " armée » " cosaque " arriva en Carnie. Cette " armée » était dirigée par le général Krassnov, un dirigeant des Cosaques du Don, qui avait combattu contre les bolcheviks, puis s'était exilé en Allemagne et ensuite en France.

Après la défaite du III ${ }^{e}$ Reich, les Cosaques se rendirent aux Britanniques, convaincus que les Occidentaux allaient se retourner contre l'URSS : si l'on en croit Magris, Krassnov, dans sa mégalomanie, espérait négocier avec le général Alexander, qui avait, comme lui, combattu les « rouges » en Courlande, et avec Churchill qui avait soutenu les Russes blancs de Dénikine ${ }^{3}$, tout comme - rappelle Magris - les Britanniques avaient

1. Claudio Magris, Illazioni su una sciabola, Pordenone, Studio Tesi, 1986, 94 p. (1 ${ }^{\text {re }}$ édition : "Rivista Milanese di economia », Cariplo, n. 9, gennaio-marzo 1984). L'édition utilisée pour cette communication est celle des Edizioni Studio Tesi.

Carlo Sgorlon, L'armata dei fiumi perduti, Milan, Mondadori, 1985, 312 p.

2. Et des ressortissants d'autres groupes ethniques caucasiens qui n’avaient jamais accepté de faire partie de l'Union soviétique. Magris parle de dix-sept groupes linguistiques et commente : « la mia relazione parla genericamente di "soldati russi anticomunisti »" (Illazioni su una sciabola, p. 18).

3. Claudio Magris, Illazioni su una sciabola, op. cit., p. 32. 
même décoré de l'ordre de l'Empire Britannique le général russe Skuro "per il suo valore nella guerra del '18- '19 contro i bolscevichi »; ce qui ne les empêcha pas, vingt-cinq ans plus tard, de remettre ce général aux Soviétiques "perché lo impiccassero " (ibid., p. 64). Donc, les Cosaques, après avoir tenté de fuir en Autriche, se rendirent aux Britanniques ; ceuxci les remirent aux Soviétiques, qui les exécutèrent, du moins leurs chefs. Mais beaucoup de Cosaques refusèrent la reddition et préférèrent se jeter dans la Drave, lestés de pierres, avec leurs armes et leurs chevaux, mais aussi avec leurs femmes et leurs enfants, car cette armée ne ressemblait pas aux armées modernes, mais plutôt aux " grandes compagnies " des XIV et $\mathrm{XV}^{\mathrm{e}}$ siècles - si ce n'est à l'armata Brancaleone : " Divisa sgargiante e sdrucita, che sembrava raffazzonata da uniforme diverse [...] difficile distinguere i gradi [...] in quell'armata sbandata, lacera e intrepida, i generali erano numerosi come i soldati e conferirsi le spalline era altrettanto facile quanto mettere la sella a un cavallo rubato ", dit Magris (ibid. p. 18), dont il faudra se rappeler l'humour.

Le point de départ est donc un fait authentique bien que peu connu, l'aventure de l'armée Krassnov, assez proche de l'épisode, plus célèbre, de l'armée Vlassov.

Pour Magris, c'est d'abord une sorte de souvenir d'enfance reconstitué : âgé de six ans, il se trouvait à Udine au moment des faits. Licia Governatori ${ }^{4}$ rappelle que, pour lui, la première élaboration littéraire de cet événement est un "elzeviro " dans le Corriere della Sera du $1^{\text {er }}$ février 1974. Cette reconstitution prend la forme d'une fiction complexe, manzonienne à certains égards, dans le bref texte narratif Illazioni su una sciabola, communément admis comme le texte qui marque, chez Magris, le passage de l'essai à la narration, roman bref plutôt que nouvelle ou récit, car il n'y a pas ici vraiment unité et unicité de la narration. La narration est la fiction d'une lettre d'un prêtre, don Guido, qui cherche à savoir si le cadavre d'un soldat cosaque tué le 2 mai 1945 dans le Val di Gorto, d'un coup de pistolet tiré contre la colonne de Cosaques en fuite vers l'Autriche, est bien celui de l'Atamàn Piotr Krassnov : ce que confirme la Pravda du 17 avril 1947, alors qu'officiellement il a été pendu à Moscou le 16 janvier 1945. Cela reste d'ailleurs la conviction de Magris, comme il le dit dans la dernière phrase du livre et le confirme dans une intervention au

4. L. Governatori, Claudio Magris, l'opera saggistica e narrativa, Trieste, Lint Editoriale Associati, 1999. 
Corriere della Sera du 13 novembre 2001. Un article du même Corriere, en date du 13 août 1957, affirmait cependant que ce corps avait été exhumé, à la demande de trois officiers allemands, et que c'était bien celui de Krassnov. Magris fait également état d'autres hypothèses : Krassnov se serait rendu aux Anglais le 27 mai 1945, et le cadavre serait celui d'un ancien officier tsariste dans la poche duquel les Cosaques auraient mis la montre de Krassnov pour faire croire à sa mort ; ou bien il se serait enfui dans la montagne en échangeant son sabre contre une poignée de vivres. Le narrateur se demande aussi pourquoi la mort de Krassnov et son enterrement ont été tenus secrets ${ }^{5}$. Il ne reconstitue pas la vérité des faits, mais le processus de leur déformation : c'est un aspect particulier de l'histoire, l'histoire de la subjectivité. Ce texte porte donc sur un doute qui ne se résout pas, comme si la vérité historique était insaisissable et qu'il n'y avait pas de véritable frontière entre vérité et mensonge ou erreur, entre vérité et fiction. Mais c'est aussi - les deux questions ne sont pas sans rapports - de la fragilité des règles morales qu'il traite. Une réflexion rendue sans doute plus aisée parce que les événements qui en sont à la base se situent en temps de guerre ; une guerre particulière, avec des renversements d'alliance ${ }^{6}$, dont les tenants et les aboutissants étaient économiques, politiques, idéologiques, mais revêtaient souvent des dimensions morales, voire religieuses, claires ou non, conscientes ou non, affichées ou non, sincères ou non, acceptables ou non.

Les deux questions ne sont effectivement pas sans rapports : vérité et mensonge ou erreur se situent sur le plan de l'incertitude du vrai, trahison ou fidélité sur celui de l'incertitude du bien ; or, la frontière entre le vrai et le bien peut parfois être bien ténue. C'est là que se situe un aspect essentiel de la question morale.

Un an après ce texte de Magris, Sgorlon publie donc L'armata dei fiumi perduti, à partir de la même référence historique, en l'intégrant d'emblée dans une fiction avouée : Questo, dit Sgorlon dans une note liminaire, è un romanzo misto di storia e d'invenzione. I personaggi sono frutto di fantasia, ma la duplice tragedia del popolo friulano e di quello cosacco appartengono agli eventi poco noti della seconda guerra mondiale " (op. cit., p. 6). Il fait de cette narration une sorte d'épopée populaire ${ }^{7}$ qui

5. Illazioni su una sciabola, op. cit., p. 22.

6. C. Sgorlon y fait allusion de façon curieuse, mettant sur le même plan, par une réflexion de Marta, les Cosaques, identifiés à des Russes qui auraient changé de camp, et les Italiens qui auraient fait de même après le 8 septembre 1943 : "Continuavano ad essere nemici, ma essendo passati dall'altra parte, perché nel frattempo, l'altra parte era mutata " (L'armata dei fiumi perduti, p. 35).

7. Comme toute l'histoire du peuple kazak selon Sgorlon. Cf., p. 288 : "Un racconto dolente, pieno di lutti e di sventure, che pareva in attesa di un poeta popolare che lo sistemasse in versi e in canzoni, e lo affidassse alla memoria del popolo, che ricorda sempre il suo passato ». 
plonge dans ses mythes habituels, la pérennité de l'histoire, la pérennité du mal, la course à la mort et au néant, mais aussi la pérennité du Frioul, de son Frioul, accoutumé à la guerre, à la peur et à la violence ${ }^{8}$ : la peur en toile de fond, et cette forme spécifique de la violence qu'est la destruction du passé, du présent, de l'avenir, notamment pour les Juifs mais aussi pour les Cosaques (ibid., p. 18), avec également son effet sur les individus ${ }^{9}$. Le roman raconte donc l'arrivée et l'installation dans un village du Frioul d'une compagnie de Cosaques. Les premiers temps sont calmes, l'atmosphère peut être parfois conviviale, même si les fêtes se font toujours aux dépens des ressources des villageois, réquisitionnées par les Cosaques : mais personne n'est dupe du mot "réquisition ", et surtout pas les Cosaques. Les villageois, d'ailleurs, prennent bientôt des mesures de rétorsion : des chevaux cosaques disparaissent, tout simplement mangés par les Frioulans affamés. Quelques intrigues et liaisons amoureuses s'esquissent ou se nouent. Marta, après avoir perdu ses amants précédents, emportés par la guerre, accueille Urvan ; Marta a une fonction particulière dans le roman qui, d'une certaine façon, tourne autour d'elle; elle n'arrive pas à voir des ennemis chez les hommes, tous victimes de la guerre, tous " poveri soldati ». Même s'il demeure longtemps à l'arrière-plan, le contexte de guerre demeure, en particulier avec la crainte, de la part des Cosaques, de l'action des partisans, qu'on ne voit pas mais dont on sait qu'ils sont là parce que, dans les villages voisins, il y a des combats, et que des Allemands et des Cosaques sont retrouvés morts dans la forêt. Certains Cosaques se doutent que les villageois sont de mèche avec les partisans, et ils traitent de plus en plus ces villageois comme des ennemis; un jour, ils violent collectivement une jeune femme de façon si brutale qu'elle en meurt. Un autre jour, ils tuent à coups de fouet un jeune homme, suspecté, à juste titre, d'être un partisan. Mais ce qui fait à la fin le fond du roman, c'est, du fait de la dégradation de la situation militaire pour les Allemands et leurs alliés, la conscience croissante qu'ont les Cosaques d'être dans une situation sans issue, de faire une guerre qui n'est pas la leur ; certains trouvent même des analogies entre leur espoir d'une terre qui soit à eux et la lutte des partisans qui veulent sauver leur indépendance. Ils finissent par s'enfuir dans la nuit et le froid et, après une ultime bataille contre les partisans, se rendent aux Anglais ; quand ils comprennent que ceux-ci les ont dupés, ils se jettent dans la rivière. Cette fin est

8. L'armata dei fiumi perduti, op. cit., p. 10 sq.

9. Voir comment la guerre a modelé Ivos : «Pareva che la persona di Ivos fosse impastata con le sue traversie di combattente e con ciò che aveva visto e vissuto sui fronti » (ibid., p. 23). 
donc conforme à l'histoire ; Sgorlon la traite sur un mode particulièrement dramatique, avec l'agonie et la mort d'un enfant cosaque malade, la violence et la trahison dans la dernière bataille, le tout dans le froid, la boue, une impression constante d'obscurité et de désespoir.

La violence est ainsi présente dans ces deux ouvrages, dénoncée et condamnée comme telle, une violence qui détruit les hommes et les femmes, l'amour, le présent, l'avenir et le passé des individus et des sociétés. Sgorlon relève aussi la violence spécifique de ce conflit, les bombardements massifs sur l'Allemagne et sur les positions cosaques ${ }^{10}$, et surtout la déportation, l'élimination des Tziganes et des Juifs (ibid., p. 8) : tout le premier chapitre est imprégné de cette violence ${ }^{11}$. Les Cosaques, dit quant à lui Magris, sont partie prenante dans cette violence de la guerre malgré leur rôle subalterne :

Venivano adibiti dai nazisti a piccole operazioni ausiliarie e odiose violenze, razzie e sequestri, venivano costretti a far la guardia delle rovine dei paesi bruciati dai tedeschi e dai fascisti [...] erano giunti in quell'angolo del mondo per costruirsi una casa e trovare riparo dall'indeterminatezza del nulla, ma avevano invece distrutto e restituito all'informe l'ordine accogliente racchiuso fra quelle mura ${ }^{12}$.

Ils ne le cèdent en rien aux nazis en matière de barbarie ${ }^{13}$, au point que certains d'entre eux ont honte de leur rôle d'envahisseurs (ibid., p. 129130).

En même temps, la violence est vue comme une fatalité historique dont la guerre est un moment symptomatique et symbolique. Elle ne s'éteint pas avec la guerre, puisque les trois officiers allemands qui viennent exhumer le corps attribué à Krassnov gardent pour eux l'or des lunettes et des dents " proprio, dit Magris, comme ai bei tempi della guerra" (ibid., p. 24).

Mais ce qui m'a paru original dans ces deux textes, c'est la place d'un aspect particulier de la violence : la trahison. Violence par ses implications concrètes, parce qu'elle est violence morale, violence faite aux rapports humains, de confiance, de convivialité même, voire de liens affectifs et amoureux. Mais aussi violence dans l'histoire : violence faite à l'histoire (la trahison de leur passé de la part des Cosaques ${ }^{14}$ ), et violence commise par l'histoire, où, on va le voir, la trahison est partout.

10. C. Sgorlon, L'armata dei fiumi perduti, p. 90-91 et 137.

11. Cf., p. 18, la destruction du patrimoine des familles juives.

12. Cl. Magris, Illazioni su una sciabola, op. cit., p. 60-61.

13. C. Sgorlon, L'armata dei fiumi perduti, op. cit., p. 208-209.

14. [Krassnov] " era arrivato lì da noi, a infangare i propri capelli bianchi opprimendo gente che non aveva mai visto, a derubarli della loro patria, che egli - proprio lui, il patriotta legittimista - voleva trasformare $[\ldots]$ nella patria cosacca $[\ldots]$ cercò la difesa dell'avventura, della cavalleria e della tradizione proprio nel nazismo, il più mortale nemico della tradizione dell'avventura, alveare totalitario e tecnolo 
Le lien entre violence et trahison est explicité en particulier dans les pages qui racontent la fin des Caucasiens où le mot «tradimento " revient deux fois chez Sgorlon (p. 283-285), alors que Magris dit que " gli Inglesi li [les Cosaques] stavano proditoriamente consegnando ai Sovietici " (p. 21).

La trahison est omniprésente dans ces deux romans, comme dans l'épisode qui les inspire. Les Soviétiques considèrent ces Cosaques comme traîtres à leur pays ${ }^{15}$ puisqu'ils ont pactisé avec les forces hitlériennes en guerre contre leur pays ; les Allemands trahissent depuis le début de l'opération ces Cosaques, envers qui ils ne tiennent pas leurs engagements : ils ne les ont jamais traités comme des alliés mais comme "gente inferiore, dei selvaggi appena domati " (Sgorlon, p. 62) qu'on achète avec des promesses fallacieuses et à qui ils n'ont jamais confié que des tâches ignobles et subalternes, au lieu de répondre à leur attente de se lancer contre les Russes, dit Sgorlon, et Magris déclare que les Cosaques « erano stati ingannati dalle promesse tedesche " (Illazioni su una sciabola, p. 49). Les Alliés trahissent, eux aussi, la confiance des Cosaques en les remettant aux Soviétiques au lieu de les engager dans une guerre de reconquête contre eux : Magris relève que

anche il maggior Davies, che recò quell'invito [à se rendre à une conférence avec le général Alexander, alors qu'il s'agissait de les rassembler pour les remettre aux Soviétiques] e se ne vergogna ancor oggi, aveva mentito ai cosacchi senza sapere, sino all'ultimo momento, di ricevere e trasmettere ordini menzogneri, e venendo tradito egli stesso. (ibid., p 50)

Krassnov trahit ses Cosaques par ses " fughe travestite da assalti e da avanzate " (ibid., p. 35). Les Cosaques, dans le roman de Sgorlon, trahissent l'hospitalité des villageois frioulans chez qui ils se sont installés et avec qui ils ont entretenu des relations cordiales; ils trahissent même leurs relations amoureuses : quand ils décident de fuir, Urvan, qui avait pourtant promis à Marta de rester avec elle, s'enfuit avec l'armée cosaque. Les villageois les trahissent, toujours chez Sgorlon, par leurs contacts avec les partisans. Dans ce contexte où la trahison est la règle générale, il n’y a pas de certitude sur qui trahit qui. Quand les Cosaques contraignent les paysans frioulans à boire avec eux, ceux-ci « si domandavano se per caso erano dei traditori perché stavano a bere con l'invasore e a far festa con lui " (L'armata dei fiumi perduti, p. 150), La trahison est le fait aussi bien d'individus que de collectivités, États ou armées.

gico che livellava la vita in una uniformità ben più ferrea di quella imputata alle disprezzate democrazie " (Cl. Magris, Illazioni su una sciabola, p. 37 et 71).

15. "imputati di alto tradimento verso il loro paese ", dit Cl. Magris (ibid., p. 49). 
L'idéologie est sans doute la composante centrale de la trahison chez les Cosaques :

Krassnov, che si voleva Atamàn di un popolo di cavalieri erranti, non capiva che i suoi pari erano quegli altri nomadi e randagi che combattevano sui monti, in difesa della loro patria, che egli, campione del patriottismo negato dall' 'internazionale rossa ed ebraica', veniva ad occupare e derubare [...] per conto terzi (Magris, p. 33).

«Krassnov fieramente illuso ed ottuso » dit encore Magris (ibid., p. 60), avec une rime intérieure qui souligne le lien entre une obstination stupide dans son choix étroitement idéologique (c'est par antisoviétisme et non par adhésion au nazisme qu'il est devenu l'allié de celui-ci) et la trahison dont il est victime.

Il y a une sorte d'engrenage de la trahison qui fait que «sono i primi passi nel male quelli di cui ci si deve guardare ». La trahison est fille du mal qui fait que la « mescolanza di colpa oggettiva e di incolpevole abbagliamento individuale [...] logora ciò che vi è di più nobile in noi e fa di ogni peccatore anzitutto una vittima, un ingannato» (ibid., p. 50) : le mal est lié à l'erreur mais se retourne contre son auteur.

La trahison est ainsi un piège pour les Cosaques dont ils ne pourraient sortir que par une nouvelle trahison : quand ils comprennent qu'ils ont été manipulés par les nazis et que cela les conduit à une catastrophe,

Urvan sentiva che era impossibile che i cosacchi cambiassero campo un'altra volta. Tutti avrebbero pensato a loro come a traditori di professione, pronti a mutar bandiera ogni volta che le cose si mettessero male. Ne andava di mezzo l'onore kazak (Sgorlon, p. 116).

L'honneur ainsi entendu conduit les Cosaques à persister dans la trahison vis-à-vis de leur pays, à l'encontre de leur nature de peuple en quête de liberté. En revanche, le pope commet cette nouvelle «trahison » : il se rend aux partisans; son sens de l'honneur, de la justice, de l'humanité, le pousse à « consegnare le armi ai patriotti di questa terra, che hanno il diritto di difenderla " (ibid., p. 235), mais Sgorlon, par la bouche d'Urvan, semble réservé sur ce qui est présenté comme une trahison: Urvan se demande si ce n'est pas la seule solution qui reste, mais il emploie le mot de "désertion». En somme, les références, les repères vitaux sont insaisissables, estompés, flous du fait de la position des Cosaques envers les nazis : ils se sentent hostiles aux « rouges " par goût de la liberté et sentent que les partisans sont hostiles aux nazis par ce même goût de la liberté : ils ont cela en commun et pourtant, du fait du jeu politique des alliances, ils sont ennemis ${ }^{16}$. Pour Magris, ce qui est décisif, au-delà des situations conjonctu-

16. On pourrait alors apporter un correctif ou un complément à la fameuse phrase de Karl von Clausewitz ("la guerre n'est rien d'autre que la continuation de la politique par d'autres moyens », in De 
relles, c'est peut-être un ensemble de valeurs morales, civiques, "politiques ». En même temps, il est lui aussi, sensibleà la situation contradictoire dans laquelle se trouvent les Cosaques, par leur choix initial, certes, mais un choix motivé par l'histoire et par leur histoire : dans L'Espresso du 13 juillet 1986, il parle de "l'enorme divario tra quello che [i cosacchi] facevano e quello che pensavano di fare [...] mescolanza di autentico e di posticcio [...] la loro vicenda che tanto più si richiamava a valori asssoluti come zar, patria, dio, terra, tanto più mostrava la sua falsità ».

Par leur statut d'écrivains d'une région de frontière, Sgorlon et Magris sont peut-être particulièrement sensibles à des questions d'identité, de patrie, de communauté, plus qu'à des questions de nation et d'Etat : les Cosaques sont en quête d'une identité recouvrée, d'une terre des pères reconstruite, en rupture avec l'Etat multinational qu'était alors l'URSS : la question de la trahison ne se pose donc sans doute pas pour eux. Sgorlon attribue la responsabilité essentielle de la guerre et de la violence aux Allemands et tend à relativiser la faute des envahisseurs cosaques, en voyant derrière eux « la fatalità degli eventi » (cf., p. 87). Magris est parfois sans indulgence pour Krassnov et son " armata collaborazionista " ( $c f$., p. 50).

Les choix narratifs de Magris sont toutefois particulièrement intéressants par l'ambiguité qu'ils introduisent dans le récit. Par exemple : « $\mathrm{Ci}$ pareva - scrive la cronaca $[. .$.$] - che i tedeschi stessero portando a termine$ un altro dei loro imbrogli ai danni dell'Atamàn " (ibid., p. 26). "Ci pareva » renvoie à l'incertitude du vrai ; « imbrogli », à la manipulation des faits, "scrive la cronaca " renvoie à un document source du document qu'est la lettre fictive de don Guido. Et c'est cette succession d'intermédiaires qui - comme dans le jeu du téléphone - est la source fondamentale de mensonges, d'erreurs, ou, pour le moins, d'ambiguïtés. Le livre se présente comme une lettre d'un prêtre, don Guido, à un ami, don Mario, à propos d'un rapport remis deux ans plus tôt à leur évêque, rapport sur une mission accomplie par don Guido auprès des troupes cosaques. On ne sait pas quelle était la destination de ce rapport (probablement « far ordine negli archivi diocesani »), mais on sait que le narrateur reprend l'enquête et écrit à don Mario après la lecture d'un article du Corriere di Trieste daté du 13 août 1957, deux ans après le rapport. C'est-à-dire que cette lettre, ce récit, intervient treize ans après les faits, après que ceux-ci sont passés par

la guerre, 1832) : pour ces Cosaques, la guerre qu'ils mènent est, bien entendu, continuation de la politique du $\mathrm{III}^{\mathrm{e}}$ Reich, mais en rupture avec leurs propres projets politiques. 
de multiples filtres narratifs. Ce prêtre lui-même peut fonctionner comme un filtre narratif: il se dit vieux, inutile, " un pensionato dello spirito ", las de vivre, las d'être chrétien, las de prier, las d'aider son prochain, las tout court. Il ne sait pas s'il a vraiment rencontré Krassnov, ne se souvient plus s'il avait on non de la barbe, etc. : « tante cose intorno a noi perdono il loro sapore [...] nella memoria che si confonde " (Magris, p. 12-13). Il n'aspire plus qu’à " andare dall'altra parte " et semble déjà se mouvoir dans l'éternité. Tout cela, y compris l'humour perceptible dans les quelques citations que je viens de faire, introduit une distance entre le récit et son référent, qui me semble au moins égale à celle des Promessi sposi.

Mais il ne faut certainement pas prendre pour argent comptant les propos de ce curé qui se présente comme au seuil de la sénilité : il dit « ho fatto ben poco ", alors qu'on lui a confié une mission diplomatique complexe et dangereuse. Magris lui donne en cachette une culture qui ne manque pas d'ouverture : on peut lire ou deviner dans ses propos (ibid., p. 14) des références à Sartre (La Nausée ${ }^{17}$ ) et à Leopardi (La quiete dopo la tempesta) $\mathrm{ou}^{18}$ à la phrase célèbre de Malraux : "Au moment où la mort change notre vie en destin ». Surtout, la qualité de sa réflexion théologique n’est pas la même que celle de don Abbondio : il se pose des questions sur le rapport entre le libre arbitre de l'homme et l'omniscience de Dieu, ce qui est presque un lieu commun de la réflexion chrétienne (cf. les Provinciales de Pascal). D'autres indices, y compris sa façon de se placer dans l'éternité (« mi sembra di muovermi liberamente nel tempo e con il tempo, dal passato verso il futuro e dal futuro verso il passato, in un presente di tutte le cose " (Magris, p. 14) ; "se le cose "sono" già nel futuro, come nel passato, forse quel loro esistere nel futuro non esclude la nostra libertà " [ibid., p. 15]) peuvent être lues à la fois comme une façon de se mettre en retrait et comme une référence à une question métaphysique qui ne relève pas seulement du dogme : l'impossibilité de concevoir l'éternité et l'infini et le lien de cette question avec celle du libre arbitre et de la prédestination (ibid., p. 40). En somme, Magris en fait un esprit ouvert, un homme intelligent, cultivé, fin, humoriste ${ }^{19}$, dont la fausse modestie montre bien qu'il

17. On a peine à imaginer qu'un écrivain de la culture de Magris n'ait pas pensé à ce passage de la Nausée : "La racine du marronnier s'enfonçait dans la terre [...] jamais, avant ces derniers jours, je n'avais pressenti ce que voulait dire "exister"..." (La Nausée, Gallimard, 1938, p. 161) en écrivant : "Non temere che voglia impelagarmi in discussioni o tirar fuori nuove prove circa l'esistenza di Dio [...] Non si dice "esistenza dell'albero"..." (Cl. Magris, Illazioni su una sciabola, p. 14).

18. Ibid., p. 22. : "Ogni morte è un mistero e, per un cristiano, è il momento decisivo della vita, in cui si dispiega la sua verità definitiva ".

19. Ne conseille-t-il pas à Dieu, pour le juger, de s'adresser à ses vieux compagnons, qu"il n’a pas su convertir, et avec qui il joue aux échecs et boit de la bière, plutôt qu'aux " sacerdoti che hanno ascoltato tutte le [sue] confessioni" (Cl. Magris, op. cit., p. 16) 
a conscience d'être tout cela. Ce qui renforce ses jugements dans leur modération : ce n'est ni la sénilité ni la faiblesse qui parle, c'est la sagesse ; le récit laisse transparaître la condamnation mais accorde une large place à la compréhension, ce qui donne peut-être plus de poids à la condamnation. Et cela nous ramène à Magris, derrière don Guido : par la série de filtres narratifs que sont les antécédents de ce récit, Magris prend du recul et dédramatise les faits tragiques auxquels il se réfere, sans les ignorer. Par exemple, dans ce livre, la violence est presque tue, elle est présente surtout indirectement, notamment par cet indice sûr, antérieur à la lettre, que le narrateur a été envoyé pour « intercedere presso i cosacchi affinché desistessero dai soprusi e dalle violenze che infliggevano a quelle povere popolazioni " (Magris, p. 11), où presque tous les mots sont des dénonciations : soprusi, violenze, infliggevano, povere popolazioni. Pour conclure avec une modestie qui laisse une impression d'humour un peu désabusé : "Dopo quella mia missione, le cose sono continuate come prima ( (ibid., p. 12).

Chez Sgorlon, il y a, au premier abord, une distanciation du même ordre. L'histoire de l'armée Krassnov est vue par les yeux de Marta, qui n'est pas neutre, car elle se place toujours du côté de ceux qui apparaissent comme des victimes. Ce qui est source d'ambiguïté, puisque l'histoire fait que Cosaques et Frioulans sont victimes les uns comme les autres, mais victimes les uns contre les autres. En fait, ils ne sont pas sujets mais objets de l'histoire : l'un des prinicpaux personnages, Gavrila, se sent " portato in quel luogo dalle correnti irresistibili della guerra [...] sbattuto lì dentro, nella situazione attuale, da una serie di fatti indefinibili, ognuno dei quali aveva determinato quello successivo ${ }^{20} "$ : il y a une succession de faits qui échappent aux hommes et qui n'obéissent pas à une logique mais sont un simple enchaînement. Un enchaînement qui va nécessairement vers la dégradation et la mort.

Une des caractéristiques stylistiques de Sgorlon est l'usage récurrent de comparaisons et métaphores parfois très expressives. Leur référent est fréquemment la mort : Urvan veut rester en vie mais « la confusione della guerra lo aveva cacciato in una sorta di binario morto " (ibid., p. 149) avec une restauration de la valeur de l'adjectif morto, perdue dans l'expression ferroviaire ; les villageois et les partisans frioulans ne pensent plus qu'à agresser les Cosaques « saltargli addosso, finirli come fossero dei cinghiali

20. L'armata dei fiumi perduti, op. cit., p 113. 
devastatori feriti a morte "(ibid., p. 233). Toutefois, par cette pratique constante de la métaphore et de la comparaison, Sgorlon finit parfois par créer un tel écart avec leur objet qu'on en vient à une perte de sens. On en a un exemple quand Gavrila prend conscience que ses espoirs de recouvrer une grande patrie cosaque étaient des rêves; Sgorlon écrit : "Le sue grandi illusioni erano state corrose in pochi mesi fino a ridursi a un guscio vuoto [...] un po' come i cadaveri che conservano un'apparenza di interezza [...] si dissolvono in una sorta di caligine nera [...] la verità era terribile, dissolvatrice come la soda caustica » (ibid., p. 138). On assiste ici à une dissolution du sens par ces comparaisons en chaîne : corrodées, coquilles vides, cadavres, lesquels sont comme de la brume noire, les illusions sont perdues de vue. Ainsi, par la tentative de donner à l'histoire une dimension qui sorte de l'humain, de la transcender, Sgorlon risque de la dépouiller de toute morale, de n'y voir ou de n'en montrer qu'une dimension dramatique existentielle et millénaire. Il voit dans ce moment contradictoire de la Seconde Guerre mondiale " un tranello della sorte " (ibid., p. 195) : l'homme, cosaque ou frioulan, est impuissant et irresponsable, face à l'histoire, à la douleur, à la mort, à l'éternité. La violence, omniprésente, sousjacente ou lointaine, est une composante essentielle, historico-génétique, de la personnalité de tout Cosaque : ils ont un goût de la guerre qui fait qu'ils se comportent exactement comme les nazis, en conquérants : désordre, violence et rapines. Quant à la guerre, elle est une forme du destin, toute-puissante, elle relève de l'éternité du mal, ce qui peut relativiser tout acte au point d'effacer toute règle morale. Les moments de paix fréquemment rappelés par Sgorlon sont tellement idylliques qu'ils semblent l'évocation d'un monde fictif, ce qui aboutit à faire de la guerre la donnée concrète, constante, normale, de l'histoire et de l'existence. La violence est un fait tellement universel qu'elle échappe à toute responsabilité individuelle, fatalité mais aussi absurdité : Ghirei se sent des affinités avec les partisans qu'il combat, des jeunes comme lui, qui " combattevano una battaglia affine a quella sognata dai cosacchi » (ibid., p. 67) ; au cours d'une patrouille solitaire, il rencontre des bergers, dont il comprend, après coup, qu'il s'agissait de partisans, avec lesquels la rencontre s'est bien passée, amicalement même, et il en est heureux : " Si era fatto abbindolare eppure era lieto che le cose fossero andate com'erano andate " (ibid., p. 160).

La généralisation de la trahison conduit aussi à estomper toute certitude morale. Quand Marta, condamnée à avoir la tête rasée pour avoir été la maîtresse d'un officier cosaque mort lors de la fuite de l'armée Krassnov, décide de se raser elle-même en signe de veuvage, ce qui devait être un 
châtiment ignominieux devient un signe qui la ramène au statut honorable de veuve de guerre.

Ce qui provoque cette généralisation de la trahison et, en même temps, la minore, c'est que c'est cette guerre elle-même qui est une trahison ; les Cosaques ont été trahis par le jeu politique auquel ils ont participé en s'alliant aux nazis, non pour faire la guerre aux Frioulans, mais pour conquérir leur liberté contre l'État soviétique. Gavrila comprend, mais un peu tard, que «non era la sua guerra, ma una tetra parodia di essa, una sagra di sfaceli luttuosi, con cui sentiva di non avere più nessun rapporto " (Sgorlon, p. 138).

On ne peut toutefois aller trop loin dans cette analyse : on n'est pas que dans la psychologie et la méta-histoire ; la "realtà effettuale delle cose ", avec les horreurs de la guerre, la déportation des Juifs et des Tziganes, plus rarement mentionnée dans la littérature, est bien présente, y compris dans les détails les plus révoltants.

Je trouve donc dans ces deux romans une ambiguïté comparable, même si les modalités et les motivations en sont quelque peu différentes : leur condamnation de la barbarie, du nazisme, du fascisme, de l'antisémitisme, est incontestable. Magris s'est exprimé à plusieurs reprises, dans les années récentes, sur ce sujet ${ }^{21}$. Cependant, les ruses et les pièges de l'histoire, le regard distancié des deux auteurs, leur vision du mal, éternel et omniprésent, sans dimension historique, pourraient revenir à donner pour inévitable, lié à l'humaine condition et au cours aventureux de l'histoire, à accepter ce que la morale est tentée, spontanément, de condamner : la violence et la trahison. C'est peut-être ici qu'intervient la réflexion de Magris sur la différence entre justice et pardon ${ }^{22}$.

21. Il s'est également prononcé clairement contre le révisionisme : " non si tratta di serene revisoni storiche, ma di una sorda apologia dei peggiori aspetti del passato " ("Dovremo ripetere no pasaran ?", Corriere della sera, 20 novembre 2002).

22. "Il perdono non può, non deve aver nulla a che fare con la giustizia [...] Il perdono riguarda la vita morale, la capacità interiore di superare stati e moti dell'animo [...] la legge [...] deve solo accertare i fatti, trovarne le eventuali aggravanti o attenuanti, qualificarli giuridicamente e applicare le relative sanzioni previste dal Codice ", in "Il perdono così facile, così vuoto ", Corriere della Sera, 31 juillet 2002. 